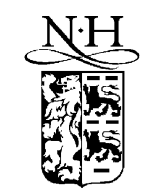

ELSEVIER

\title{
Error estimates for an operator-splitting method for incompressible flows
}

\author{
J. Blasco ${ }^{\mathrm{a}, *}$, R. Codina ${ }^{\mathrm{b}}$ \\ a Departament de Matemàtica Aplicada I, ETSEIB, Universitat Politècnica de Catalunya, Campus Sud, Edifici H, \\ Avgda. Diagonal 647, 08028 Barcelona, Spain \\ b Departament de Resistència de Materials i Estructures a l'Enginyeria, ETSECCPB, Universitat Politècnica de Catalunya, \\ Campus Nord, Edifici C1, c/Jordi Girona 1-3, 08034 Barcelona, Spain
}

Available online 16 March 2004

\begin{abstract}
In this paper we provide an error analysis of a fractional-step method for the numerical solution of the incompressible Navier-Stokes equations. Under mild regularity assumptions on the continuous solution, we obtain first order error estimates in the time step size, both for the intermediate and the end-of-step velocities of the method; we also give some error estimates for the pressure solution.
\end{abstract}

๑ 2004 IMACS. Published by Elsevier B.V. All rights reserved.

Keywords: Incompressible viscous flow; Navier-Stokes equations; Fractional-step methods; Finite elements; Error analysis

\section{Introduction}

The numerical solution of the unsteady, incompressible Navier-Stokes equations has received much attention in the last decades, and many numerical schemes are now available for that purpose. The difficulties encountered in this problem are mainly of three different kinds: the mixed type of the equations, which is due to the coupling of the momentum equation with the incompressibility condition, and, subsequently, the treatment of the pressure; the advective-diffusive character of the equations, which have a viscous and a convective term; and finally, the nonlinearity of the problem.

Fractional-step methods are becoming widely used in this context. By splitting the time advancement into a number of (generally two) substeps, they allow to separate the effects of the different operators

\footnotetext{
* Corresponding author.

E-mail addresses: jorge.blasco@upc.es (J. Blasco), ramon.codina@upc.es (R. Codina).

URLs: http://www-ma1.upc.es/ blasco (J. Blasco), http://www.rmee.upc.es/homes/codina (R. Codina).
} 
appearing in the equations (see, for instance, [3]). They have been used together with different space discretizations: finite difference [4,14,15], finite element [8,17] and spectral element methods [26]. However, semidiscrete presentations of these methods, in which the space variables are not discretized, seem more appropriate to study the effect of the time discretization itself.

The origin of this category of methods is generally credited to the work of Chorin [4] and Temam [22]. They developed the well-known projection method, which is a two step method in which the second step consists of the projection of an intermediate velocity field onto the space of solenoidal vector fields, thus enforcing incompressibility. The incompatibility of the projection boundary conditions with those of the original problem may introduce a numerical boundary layer of size $\mathrm{O}(\sqrt{v \delta t})$ in these methods $[18,25]$, where $v$ is the kinematic viscosity and $\delta t$ is the time step size. However, convergence of this method to a continuous solution as $\delta t$ tends to zero was proved in [23], for the semidiscrete method, and [5], for a fully discrete method with periodic boundary conditions. The end-of-step velocities of the projection method do not converge in the space $\mathbf{H}_{0}^{1}(\Omega)$, since they do not satisfy the correct boundary conditions.

More recently, analytical studies of fractional step methods have turned into obtaining error estimates in the time step size, so as to establish their order of accuracy. Thus, Shen proved in [20] that the projection method, both with and without pressure correction, is first order accurate in a certain norm. A more recent analysis given in [12] for a fully discrete, finite element implementation of the incremental fractional step projection orden in the mesh size, First order error estimates fractional step method, from incompressibility.
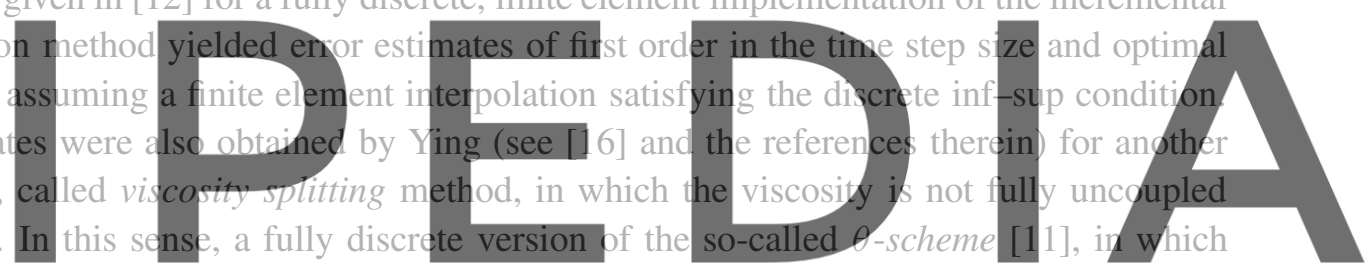

viscosity and incompressibility are also coupled, was proved to converge to a continuous solution in [9]

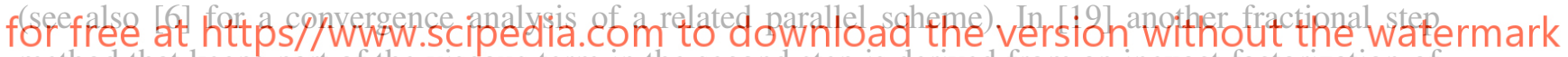
method that keeps part of the viscous term in the second step is derived from an inexact factorization of the fully discrete original problem; this method is referred to as Yosida scheme in this reference.

In this paper we provide some error estimates for an operator splitting, fractional step method which was introduced and studied in [1]. It is a two step scheme in which the nonlinearity and the incompressibility of the problem are split into different steps. It allows to enforce the original boundary conditions of the problem in all substeps of the scheme, which leads to convergence of both the intermediate and the end-of-step velocities of the method to a continuous solution in the spaces $\mathbf{L}^{2}(\Omega)$ and $\mathbf{H}_{0}^{1}(\Omega)$ (see [1]). Here we prove that these velocities are first order accurate in the time step size.

Moreover, the study of this method was originally motivated by the consideration of a well-known predictor-multicorrector algorithm (see [2]), as detailed in [1]; this fact provides a theoretical explanation of why the original boundary conditions of the problem can be prescribed in this algorithm, and in what sense it can be understood as a fractional step method.

The paper is organized as follows: in Section 2 we introduce the notation we use and some generalities about the incompressible Navier-Stokes equations, such as the regularity assumed for their solutions. In Section 3 we recall the fractional step method of [1] and introduce a finite element spatial approximation, while in Section 4 we give an error analysis of this method; we first obtain some error estimates for both the intermediate and the end-of-step velocities and then analyze the pressure solution. 


\section{Preliminaries}

The evolution of viscous, incompressible fluid flow in a bounded domain $\Omega \subset \mathbb{R}^{d}(d=2,3)$ is governed, in the primitive variable formulation, by the unsteady, incompressible Navier-Stokes equations:

$$
\begin{aligned}
& \frac{\partial \boldsymbol{u}}{\partial t}+(\boldsymbol{u} \cdot \nabla) \boldsymbol{u}-v \Delta \boldsymbol{u}+\nabla p=\boldsymbol{f} \text { in } \Omega \times(0, T), \\
& \nabla \cdot \boldsymbol{u}=0 \quad \text { in } \Omega \times(0, T), \\
& u=0 \quad \text { on } \partial \Omega \times(0, T), \\
& u=u^{0} \quad \text { in } \Omega \times\{0\},
\end{aligned}
$$

where $\boldsymbol{u}(\boldsymbol{x}, t) \in \mathbb{R}^{d}$ is the fluid velocity at position $\boldsymbol{x} \in \Omega$ and time $t \in(0, T)$ (with $T>0$ given), $p(\boldsymbol{x}, t) \in \mathbb{R}$ is the fluid kinematic pressure, $v>0$ is the kinematic viscosity (which is assumed constant), $\boldsymbol{f}(\boldsymbol{x}, t)$ is an external force term, $\nabla$ is the gradient operator, $\nabla \cdot$ is the divergence operator and $\Delta$ is the Laplacian operator (here, and in what follows, boldface characters denote vector quantities). We consider only the homogeneous Dirichlet type boundary condition (3) for the sake of simplicity.

In order to study approximation schemes for this problem, we first introduce some notation. denote by $(\cdot, \cdot)$ the scalar product in $L^{2}(\Omega)$, and by $\|u\|_{0}=(u, u)^{1 / 2}$ its norm; the quotient $L_{0}^{2}(\Omega)=L^{2}(\Omega) / \mathbb{R}$ is needed in the case of Dirichlat type boundary then determined only up to an additive constant; moreover, given $m$ $H^{m}(\Omega)$ are denoted by $(u, v)_{m}$ and $\|u\|_{m}$, respectively. If $\mathcal{D}(\Omega)$

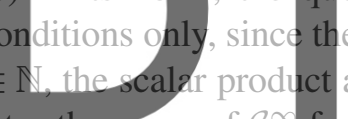

compact support in $\Omega$, then $H_{0}^{1}(\Omega)$ is the closure of $\mathcal{D}(\Omega)$ in $H^{1}(\Omega)$; the Poincaré-Friedrich inequality

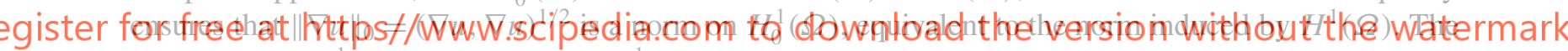
dual space of $H_{0}^{1}(\Omega)$ is denoted by $H^{-1}(\Omega)$ with norm $\|\cdot\|_{-1}$, the duality pairing between these spaces being denoted by $\langle$,$\rangle . All these definitions carry over to d$-dimensional vector valued function spaces.

Due to the incompressibility condition (2), closed subspaces of solenoidal vector fields of these Hilbert spaces are also needed. Thus, we define:

$$
\begin{aligned}
& H=\left\{\boldsymbol{u} \in \mathbf{L}^{2}(\Omega) / \nabla \cdot \boldsymbol{u}=0, \boldsymbol{n} \cdot \boldsymbol{u}_{\mid \partial \Omega}=0\right\}, \\
& V=\left\{\boldsymbol{u} \in \mathbf{H}_{0}^{1}(\Omega) / \nabla \cdot \boldsymbol{u}=0\right\} .
\end{aligned}
$$

In this notation, assuming $\boldsymbol{f} \in L^{2}\left(0, T ; \mathbf{H}^{-1}(\Omega)\right)$ and $\boldsymbol{u}_{0} \in H$ problem (1)-(4) has at least one solution $(\boldsymbol{u}, p)$ which satisfies $\boldsymbol{u} \in L^{\infty}(0, T ; H) \cap L^{2}(0, T ; V)$ (see [24]). Uniqueness and more regularity of the solution can also be proved by strengthening the assumptions on the data. In particular, we will assume that $\boldsymbol{u}$ and $p$ satisfy:

(R1) $\boldsymbol{u} \in \mathcal{C}^{0}(0, T ; H) \cap L^{\infty}\left(0, T ; \mathbf{H}^{2}(\Omega)\right), \nabla p \in L^{\infty}\left(0, T ; \mathbf{L}^{2}(\Omega)\right)$,

(R2a) $\boldsymbol{u}_{t} \in L^{2}\left(0, T ; \mathbf{L}^{2}(\Omega)\right)$,

(R2b) $\boldsymbol{u}_{t} \in L^{2}\left(0, T ; \mathbf{H}_{0}^{1}(\Omega)\right)$,

(R3) $\sqrt{t} \boldsymbol{u}_{t t} \in L^{2}\left(0, T ; \mathbf{H}^{-1}(\Omega)\right)$, 
so that we will assume either (R2a) of (R2b) depending on the context (the subindex $t$ is employed hereafter for $\partial / \partial t$ and $V^{\prime}$ stands for the dual space of $V$ ). Conditions (R1) and (R2b) can be proved, for instance, assuming that:

$$
\boldsymbol{u}_{0} \in \mathbf{H}^{2}(\Omega) \cap V, \quad f \in L^{\infty}(0, T ; H), \quad \boldsymbol{f}_{t} \in L^{1}(0, T ; H)
$$

and, if $d=3$, a condition relating $v, \boldsymbol{f}, \boldsymbol{u}_{0}$ and $T$ (namely, [24, 3.115, p. 304]), when $\Omega$ is of class $\mathcal{C}^{2}$ or is a convex polygon or polyhedron (see [24, Theorem 3.7, p. 303 and Theorem 3.8, p. 306]); under these assumptions, (R3) follows from [13, Theorem 2.3, pp. 284-285]. These results also hold when $\Omega$ is a convex polygon, since some of them rely on the additional regularity of solutions of the Stokes problem in $\Omega$ with right side in $\mathbb{L}^{2}(\Omega)$, and are also generally believed to hold on a convex polyhedron (see [13] and the references therein). Furthermore, we will also assume (see $[20,21])$ that:

\section{(R4) $\boldsymbol{u}_{t t} \in L^{2}\left(0, T ; V^{\prime}\right)$,}

a condition which requires some nonlocal compatibility conditions.

Error analysis of time integration schemes for time-dependent partial differential equations are usually given in terms of the following norms: given a Banach space W with nom $\|$ - $\mid$ w, a continuous function $u:[0, T] \rightarrow W$ and two reat $n=0, \ldots, M=[T / \delta t] ;$ a of $u$ in $l^{p}(W)$ if there exists a constant $C$ sueh that,
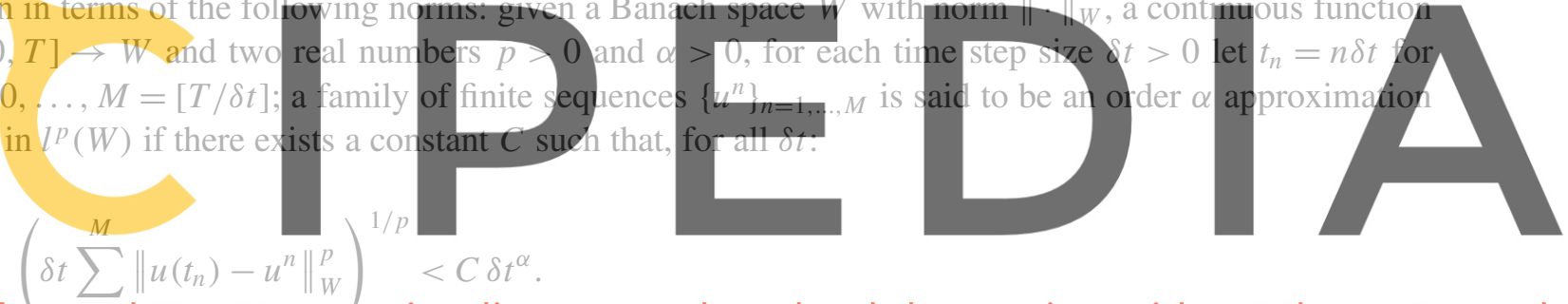

egister for free att https//www.scipedia.com to download the version without the watermark

Moreover, $\left\{u^{n}\right\}_{n=1, \ldots, M}$ is an order $\alpha$ approximation of $u$ in $l^{\infty}(W)$ if:

$$
\left\|u\left(t_{n}\right)-u^{n}\right\|_{W}<C \delta t^{\alpha}, \quad \forall n=1, \ldots, M
$$

Here, and in what follows, $C$ denotes a generic constant, possibly different at different occurrences, which may depend on the data $\boldsymbol{f}, \boldsymbol{u}_{0}, T$ and $v$, the domain $\Omega$ and the continuous solution $\boldsymbol{u}$, but is independent of the time step $\delta t$ and the mesh size $h$.

For the treatment of the convective term in the momentum equation (1), the following trilinear form is usually considered:

$$
c(\boldsymbol{u}, \boldsymbol{v}, \boldsymbol{w})=((\boldsymbol{u} \cdot \nabla) \boldsymbol{v}, \boldsymbol{w}), \quad \forall \boldsymbol{u} \in \mathbf{H}^{1}(\Omega), \boldsymbol{v} \in \mathbf{H}^{1}(\Omega), \boldsymbol{w} \in \mathbf{H}_{0}^{1}(\Omega)
$$

This form is well defined and continuous on these spaces (see [24]), and it is skew-symmetric in its last two arguments if $\boldsymbol{u} \in H$, that is, if $\nabla \cdot \boldsymbol{u}=0$ and $\boldsymbol{n} \cdot \boldsymbol{u}=0$ :

$$
c(\boldsymbol{u}, \boldsymbol{v}, \boldsymbol{v})=0, \quad \forall \boldsymbol{u} \in H, \boldsymbol{v} \in \mathbf{H}_{0}^{1}(\Omega)
$$


Moreover, $c$ has some continuity properties which hold when $\Omega$ is regular enough (see [7]) and which we will use in our proofs, such as:

$$
c(\boldsymbol{u}, \boldsymbol{v}, \boldsymbol{w}) \leqslant C\left\{\begin{array}{l}
\|\boldsymbol{u}\|_{0}\|\boldsymbol{v}\|_{2}\|\boldsymbol{w}\|_{1}, \\
\|\boldsymbol{u}\|_{0}\|\boldsymbol{v}\|_{1}\|\boldsymbol{w}\|_{2}, \\
\|\boldsymbol{u}\|_{2}\|\boldsymbol{v}\|_{1}\|\boldsymbol{w}\|_{0}, \\
\|\boldsymbol{u}\|_{0}^{1 / 2}\|\boldsymbol{u}\|_{1}^{1 / 2}\|\boldsymbol{v}\|_{1}\|\boldsymbol{w}\|_{1}, \\
\|\boldsymbol{u}\|_{1}\|\boldsymbol{v}\|_{1}\|\boldsymbol{w}\|_{0}^{1 / 2}\|\boldsymbol{w}\|_{1}^{1 / 2}, \\
\|\boldsymbol{u}\|_{1}\|\boldsymbol{v}\|_{1}\|\boldsymbol{w}\|_{L^{3}(\Omega)}, \\
\|\boldsymbol{u}\|_{L^{8}(\Omega)}\|\boldsymbol{v}\|_{1}\|\boldsymbol{w}\|_{L^{8 / 3}(\Omega)} .
\end{array}\right.
$$

Although this form is suitable for our analysis of the semidiscrete method, we will use the skewsymmetric part of $c$ in the fully discrete problem, since incompressibility is only enforced weakly in the discrete setting; thus, we define:

$$
\tilde{c}(\boldsymbol{u}, \boldsymbol{v}, \boldsymbol{w})=(1 / 2)(c(\boldsymbol{u}, \boldsymbol{v}, \boldsymbol{w})-c(\boldsymbol{u}, \boldsymbol{w}, \boldsymbol{v})), \quad \forall \boldsymbol{u} \in \mathbf{H}^{1}(\Omega), \boldsymbol{v} \in \mathbf{H}_{0}^{1}(\Omega), \boldsymbol{w} \in \mathbf{H}_{0}^{1}(\Omega) .
$$

Obviously, this form retains the continuity properties of the original form $c$ (but for the fifth one), and is skew-symmetric in its last two arguments for any $u \in \mathbb{H}^{1}(\Omega)$.

In some of our proofs we will also make yse of the operator operator $A=-P_{H} \Delta, P_{H}$ being the projection onto $H$. The latter is defi and is an unbounded, positive, self-adjoint closed operator onto $H$ $v=A^{-1} u$ is the solution of the following Stokes problem:

$$
-\Delta v+\nabla r=u \quad \text { in } \Omega,
$$

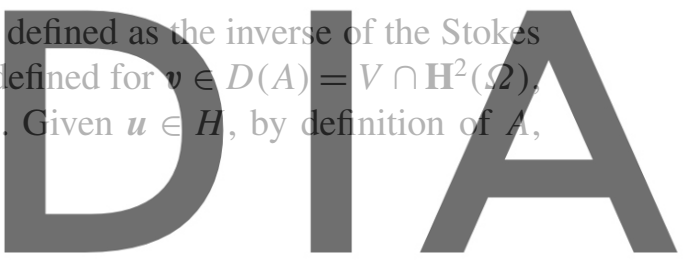

$\nabla \cdot v=0 \quad$ in $\Omega$,

egister for free at https//www ws scipedia.com to download the version without the watgermark

When $\Omega$ is of class $C^{2}$, or is a convex polygon or polyhedron (see [13]), there exists a constant $C_{1}>0$ such that:

$$
\left\|A^{-1} \boldsymbol{u}\right\|_{s} \leqslant C_{1}\|\boldsymbol{u}\|_{s-2}, \quad \text { for } s=1,2 .
$$

Furthermore, from (6) one gets $\left(A^{-1} \boldsymbol{u}, \boldsymbol{u}\right)=\left\|A^{-1} \boldsymbol{u}\right\|_{1}$, and then it is easily seen that:

$$
\|\boldsymbol{u}\|_{V^{\prime}}^{2}=\left(A^{-1} \boldsymbol{u}, \boldsymbol{u}\right)
$$

for all $\boldsymbol{u} \in H$. We will use these results in what follows.

\section{Fractional-step method}

The fractional-step method we analyze here was introduced in [1], where stability and convergence, both in the spaces $L^{\infty}\left(0, T ; \mathbf{L}^{2}(\Omega)\right)$ and $L^{2}\left(0, T ; \mathbf{H}_{0}^{1}(\Omega)\right)$ and of both the intermediate and the end-ofstep velocities, where proved. Given $\boldsymbol{u}^{n} \in V$, approximation of $\boldsymbol{u}$ at $t=t_{n}$, the time advancement to $t_{n+1}$ is split into two steps: 
First step. The first step of the method, which includes viscous and convective effects, consists of finding an intermediate velocity $\boldsymbol{u}^{n+1 / 2}$ such that:

$$
\begin{aligned}
& \frac{\boldsymbol{u}^{n+1 / 2}-\boldsymbol{u}^{n}}{\delta t}-v \Delta \boldsymbol{u}^{n+1 / 2}+\left(\boldsymbol{u}^{n} \cdot \nabla\right) \boldsymbol{u}^{n+1 / 2}=\boldsymbol{f}\left(t_{n+1}\right), \\
& \left.\boldsymbol{u}^{n+1 / 2}\right|_{\partial \Omega}=0 .
\end{aligned}
$$

Second step. Given $\boldsymbol{u}^{n+1 / 2}$ from (9), (10), the second step of the method consists of finding $\boldsymbol{u}^{n+1}$ and $p^{n+1}$ such that:

$$
\begin{aligned}
& \frac{\boldsymbol{u}^{n+1}-\boldsymbol{u}^{n+1 / 2}}{\delta t}-v \Delta\left(\boldsymbol{u}^{n+1}-\boldsymbol{u}^{n+1 / 2}\right)+\nabla p^{n+1}=0, \\
& \nabla \cdot u^{n+1}=0, \\
& \left.u^{n+1}\right|_{\partial \Omega}=0 .
\end{aligned}
$$

As can be observed in (11), the main difference between this method and the standard projection method is the introduction of a viscous term in the incompressibility step, which allows the imposition of the original boundary condition (13) on the end-of-step velocity $\boldsymbol{u}^{n+1}$. Similar ideas can be found in the $\theta$-method of Glowinski and others (see [11], for instance) and in several other methods such as those of $[6,16,17]$ or $[26]$, all of $w$ observed in (9), (10) and are the two main difficulties convective term, although The motivations that led us to the be used to explain theoretically a class of predictor
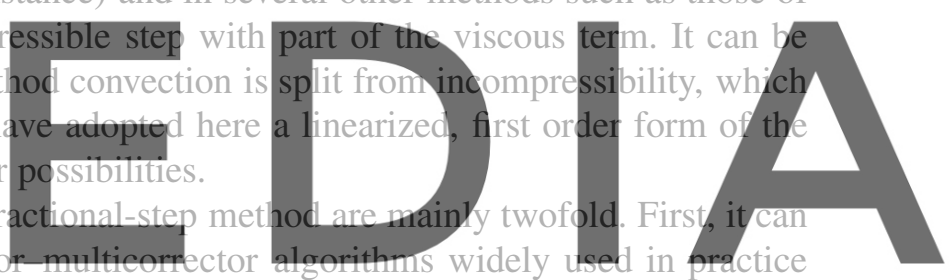

(see [1] for a more detailed explanation). These methods are based on an iterative scheme consisting

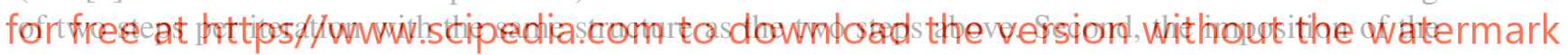
original boundary conditions on the end-of-step velocity. It is common practice among some users of the classical projection method to enforce all the boundary conditions on this field, although this is in principle not allowed if the viscous term in Eq. (11) is dropped. The present scheme, however, is not subject to this controversy; moreover, the fact that $\boldsymbol{u}^{n+1}$ satisfies the correct boundary conditions led to improved convergence results in [1] with respect to those known for that variable in the standard projection method, and will allow us to obtain improved error estimates here too.

The computational efficiency of the scheme (9)-(13) was studied in [1]. The first step of the method, which is a linear, elliptic problem, can be seen as a linearized Burger's problem; on the other hand, the second step has the structure of a Stokes (mixed) problem, the discretization of which leads to a symmetric system of linear equations. Based on ideas taken from the predictor-multicorrector algorithm used in [2], we developed in [1] an iterative technique for the solution of these two problems, in which each iteration consists of the solution of two linear systems with a diagonal matrix and a system with a symmetric, positive (semi)definite matrix, which is the same for all iterations and time steps (and thus needs being computed and factorized only once at the beginning of the calculations); this iteration showed good convergence results in several test cases, which makes the present fractional-step method feasible from a practical viewpoint. One drawback of this method is the need for the spatial discretization used to satisfy the discrete inf-sup compatibility condition, something which is nowadays known to apply to most versions of the standard projection method too (see [12]). 


\section{Error analysis}

We present here an error analysis of the fractional-step method introduced in the previous section. Although we consider the first order, linearized form of the convective term $\left(\boldsymbol{u}^{n} \cdot \nabla\right) \boldsymbol{u}^{n+1 / 2}$, similar error estimates can be obtained for other approaches, such as the fully nonlinear form $\left(\boldsymbol{u}^{n+1 / 2} \cdot \nabla\right) \boldsymbol{u}^{n+1 / 2}$; likewise, other approximations of the viscous term than the backward Euler method used here, such as the trapezoidal rule, could also be studied.

\subsection{Error estimates for the semidiscrete velocities}

Let us define the semidiscrete velocity errors as:

$$
\begin{aligned}
& \boldsymbol{e}_{c}^{n+1}=\boldsymbol{u}\left(t_{n+1}\right)-\boldsymbol{u}^{n+1}, \\
& \boldsymbol{e}_{c}^{n+1 / 2}=\boldsymbol{u}\left(t_{n+1}\right)-\boldsymbol{u}^{n+1 / 2},
\end{aligned}
$$

where the subseript $c$ refers to the fact that the space variables remain 'continuous'. We give a first estimate for $\boldsymbol{e}_{c}^{n+1}$ and $\boldsymbol{e}_{c}^{n+1 / 2}$ which shows that both $\boldsymbol{u}^{n+1}$ and $\boldsymbol{u}^{n+1 / 2}$ are order $1 / 2$ approximations to $\boldsymbol{u}$ in $l^{\infty}\left(\mathbf{L}^{2}(\Omega)\right)$ and in $l^{2}\left(\mathbf{H}_{0}^{1}(\Omega)\right)$ :

\section{Lemma 1. Assume that (R1), (R2a) and (R3) hold; then for $\mathrm{N}=0$
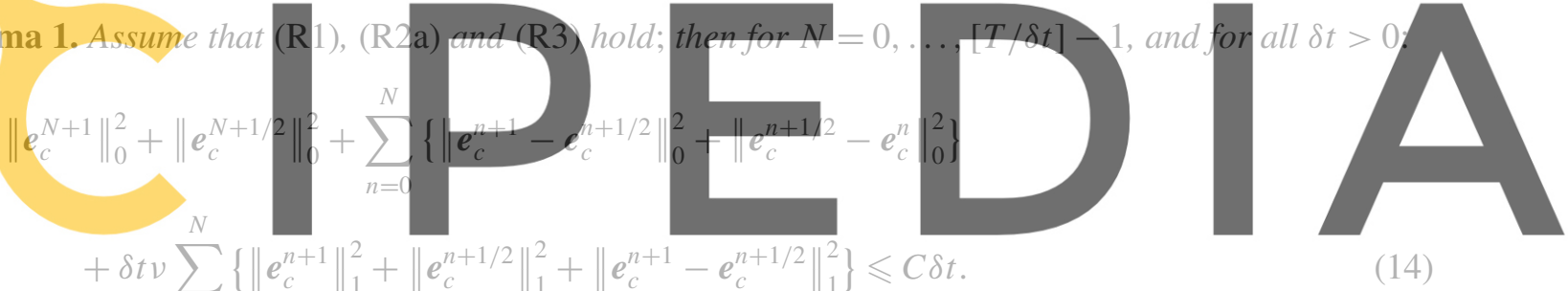 \\ (14)}
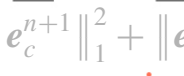

\section{egister for free at https//Www.scipedia.com to download the version without the watermark}

Proof. The first part of the proof is similar to that of [20]. We call $\mathbb{R}^{n}$ the truncation error defined by:

$$
\frac{1}{\delta t}\left(\boldsymbol{u}\left(t_{n+1}\right)-\boldsymbol{u}\left(t_{n}\right)\right)-v \Delta\left(\boldsymbol{u}\left(t_{n+1}\right)\right)+\left(\boldsymbol{u}\left(t_{n+1}\right) \cdot \nabla\right) \boldsymbol{u}\left(t_{n+1}\right)+\nabla p\left(t_{n+1}\right)=\boldsymbol{f}\left(t_{n+1}\right)+\mathbf{R}^{n},
$$

so that

$$
\mathbf{R}^{n}=\frac{1}{\delta t} \int_{t_{n}}^{t_{n+1}}\left(t-t_{n}\right) \boldsymbol{u}_{t t}(t) \mathrm{d} t .
$$

Subtracting (9) from (15), we get:

$$
\frac{1}{\delta t}\left(\boldsymbol{e}_{c}^{n+1 / 2}-\boldsymbol{e}_{c}^{n}\right)-v \Delta\left(\boldsymbol{e}_{c}^{n+1 / 2}\right)=\left(\boldsymbol{u}^{n} \cdot \nabla\right) \boldsymbol{u}^{n+1 / 2}-\left(\boldsymbol{u}\left(t_{n+1}\right) \cdot \nabla\right) \boldsymbol{u}\left(t_{n+1}\right)+\mathbf{R}^{n}-\nabla p\left(t_{n+1}\right) .
$$

We take the inner product of (16) with $2 \delta t \boldsymbol{e}_{c}^{n+1 / 2}$, use the identity $(a-b, 2 a)=|a|^{2}-|b|^{2}+|a-b|^{2}$ and split the nonlinear terms on the right side of (16) as:

$$
\begin{aligned}
& \left(\boldsymbol{u}^{n} \cdot \nabla\right) \boldsymbol{u}^{n+1 / 2}-\left(\boldsymbol{u}\left(t_{n+1}\right) \cdot \nabla\right) \boldsymbol{u}\left(t_{n+1}\right) \\
& \quad=-\left(\boldsymbol{e}_{c}^{n} \cdot \nabla\right) \boldsymbol{u}^{n+1 / 2}+\left(\left(\boldsymbol{u}\left(t_{n}\right)-\boldsymbol{u}\left(t_{n+1}\right)\right) \cdot \nabla\right) \boldsymbol{u}^{n+1 / 2}-\left(\boldsymbol{u}\left(t_{n+1}\right) \cdot \nabla\right) \boldsymbol{e}_{c}^{n+1 / 2},
\end{aligned}
$$


to obtain:

$$
\begin{aligned}
& \left\|\boldsymbol{e}_{c}^{n+1 / 2}\right\|_{0}^{2}-\left\|\boldsymbol{e}_{c}^{n}\right\|_{0}^{2}+\left\|\boldsymbol{e}_{c}^{n+1 / 2}-\boldsymbol{e}_{c}^{n}\right\|_{0}^{2}+2 \delta t v\left\|\boldsymbol{e}_{c}^{n+1 / 2}\right\|_{1}^{2} \\
& =2 \delta t\left\langle\mathbf{R}^{n}, \boldsymbol{e}_{c}^{n+1 / 2}\right\rangle-2 \delta t\left(\nabla p\left(t_{n+1}\right), \boldsymbol{e}_{c}^{n+1 / 2}\right)-2 \delta t c\left(\boldsymbol{e}_{c}^{n}, \boldsymbol{u}^{n+1 / 2}, \boldsymbol{e}_{c}^{n+1 / 2}\right) \\
& \quad+2 \delta t c\left(\boldsymbol{u}\left(t_{n}\right)-\boldsymbol{u}\left(t_{n+1}\right), \boldsymbol{u}^{n+1 / 2}, \boldsymbol{e}_{c}^{n+1 / 2}\right)-2 \delta t c\left(\boldsymbol{u}\left(t_{n+1}\right), \boldsymbol{e}_{c}^{n+1 / 2}, \boldsymbol{e}_{c}^{n+1 / 2}\right) .
\end{aligned}
$$

We bound each term in the RHS of (18) independently:

- Taylor residual term:

$$
\begin{aligned}
& 2 \delta t\left\langle\mathbb{R}^{n}, e_{c}^{n+1 / 2}\right\rangle \leqslant \frac{\delta t v}{3}\left\|e_{c}^{n+1 / 2}\right\|_{1}^{2}+\frac{C}{\delta t}\left\|\int_{t_{n}}^{t_{n+1}}\left(t-t_{n}\right) u_{t t} \mathrm{~d} t\right\|_{-1}^{2} \\
& \leqslant \frac{\delta t v}{3}\left\|e_{c}^{n+1 / 2}\right\|_{1}^{2}+C \delta t \int_{t_{n}}^{t_{n+1}} t\left\|\boldsymbol{u}_{t t}\right\|_{-1}^{2} \mathrm{~d} t
\end{aligned}
$$

- Pressure gradient term:
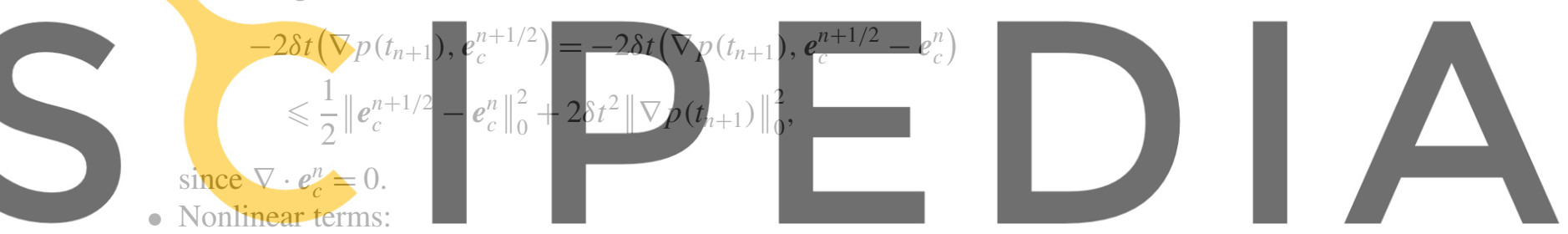

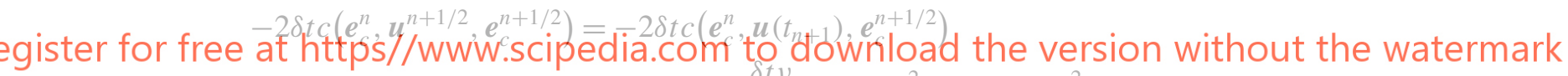

$$
\begin{aligned}
& \leqslant C \delta t\left\|e_{c}^{n}\right\|_{0}\left\|u\left(t_{n+1}\right)\right\|_{2}\left\|e_{c}^{n+1 / 2}\right\|_{1} \leqslant \frac{\delta t v}{3}\left\|e_{c}^{n+1 / 2}\right\|_{1}^{2}+C \delta t\left\|e_{c}^{n}\right\|_{0}^{2}, \\
& 2 \delta t c\left(\boldsymbol{u}\left(t_{n}\right)-\boldsymbol{u}\left(t_{n+1}\right), \boldsymbol{u}^{n+1 / 2}, \boldsymbol{e}_{c}^{n+1 / 2}\right) \\
& =2 \delta t c\left(\boldsymbol{u}\left(t_{n}\right)-\boldsymbol{u}\left(t_{n+1}\right), \boldsymbol{u}\left(t_{n+1}\right), \boldsymbol{e}_{c}^{n+1 / 2}\right) \\
& \leqslant C \delta t\left\|\boldsymbol{u}\left(t_{n}\right)-\boldsymbol{u}\left(t_{n+1}\right)\right\|_{0}\left\|\boldsymbol{u}\left(t_{n+1}\right)\right\|_{2}\left\|\boldsymbol{e}_{c}^{n+1 / 2}\right\|_{1} \\
& \leqslant \frac{\delta t v}{3}\left\|\boldsymbol{e}_{c}^{n+1 / 2}\right\|_{1}^{2}+C \delta t^{2} \int_{t_{n}}^{t_{n+1}}\left\|\boldsymbol{u}_{t}\right\|_{0}^{2} \mathrm{~d} t-2 \delta t c\left(\boldsymbol{u}\left(t_{n+1}\right), \boldsymbol{e}_{c}^{n+1 / 2}, \boldsymbol{e}_{c}^{n+1 / 2}\right)=0,
\end{aligned}
$$

where we have used (R1) and the continuity and skew-symmetry properties of the trilinear form $c$. From all these inequalities we deduce:

$$
\begin{aligned}
& \left\|\boldsymbol{e}_{c}^{n+1 / 2}\right\|_{0}^{2}-\left\|\boldsymbol{e}_{c}^{n}\right\|_{0}^{2}+\frac{1}{2}\left\|\boldsymbol{e}_{c}^{n+1 / 2}-\boldsymbol{e}_{c}^{n}\right\|_{0}^{2}+\delta t v\left\|\boldsymbol{e}_{c}^{n+1 / 2}\right\|_{1}^{2} \\
& \leqslant C \delta t \int_{t_{n}}^{t_{n+1}} t\left\|\boldsymbol{u}_{t t}\right\|_{-1}^{2} \mathrm{~d} t+C \delta t^{2} \int_{t_{n}}^{t_{n+1}}\left\|\boldsymbol{u}_{t}\right\|_{0}^{2} \mathrm{~d} t+2 \delta t^{2}\left\|\nabla p\left(t_{n+1}\right)\right\|_{0}^{2}+C \delta t\left\|\boldsymbol{e}_{c}^{n}\right\|_{0}^{2} .
\end{aligned}
$$


The proof is now different from that of [20]. We rewrite (11) as:

$$
\frac{\boldsymbol{e}_{c}^{n+1}-\boldsymbol{e}_{c}^{n+1 / 2}}{\delta t}-v \Delta\left(\boldsymbol{e}_{c}^{n+1}-\boldsymbol{e}_{c}^{n+1 / 2}\right)-\nabla p^{n+1}=0 .
$$

Taking the inner product of (20) with $2 \delta t \boldsymbol{e}_{c}^{n+1}$, we get, given that $\nabla \cdot \boldsymbol{e}_{c}^{n+1}=0$ :

$$
\begin{aligned}
\| \boldsymbol{e}_{c}^{n+1} & \left\|_{0}^{2}-\right\| \boldsymbol{e}_{c}^{n+1 / 2}\left\|_{0}^{2}+\right\| \boldsymbol{e}_{c}^{n+1}-\boldsymbol{e}_{c}^{n+1 / 2} \|_{0}^{2} \\
& +\delta t v\left(\left\|\boldsymbol{e}_{c}^{n+1}\right\|_{1}^{2}-\left\|\boldsymbol{e}_{c}^{n+1 / 2}\right\|_{1}^{2}+\left\|\boldsymbol{e}_{c}^{n+1}-\boldsymbol{e}_{c}^{n+1 / 2}\right\|_{1}^{2}\right)=0 .
\end{aligned}
$$

Adding up (19) and (21) for $n=0, \ldots, N$, we find:
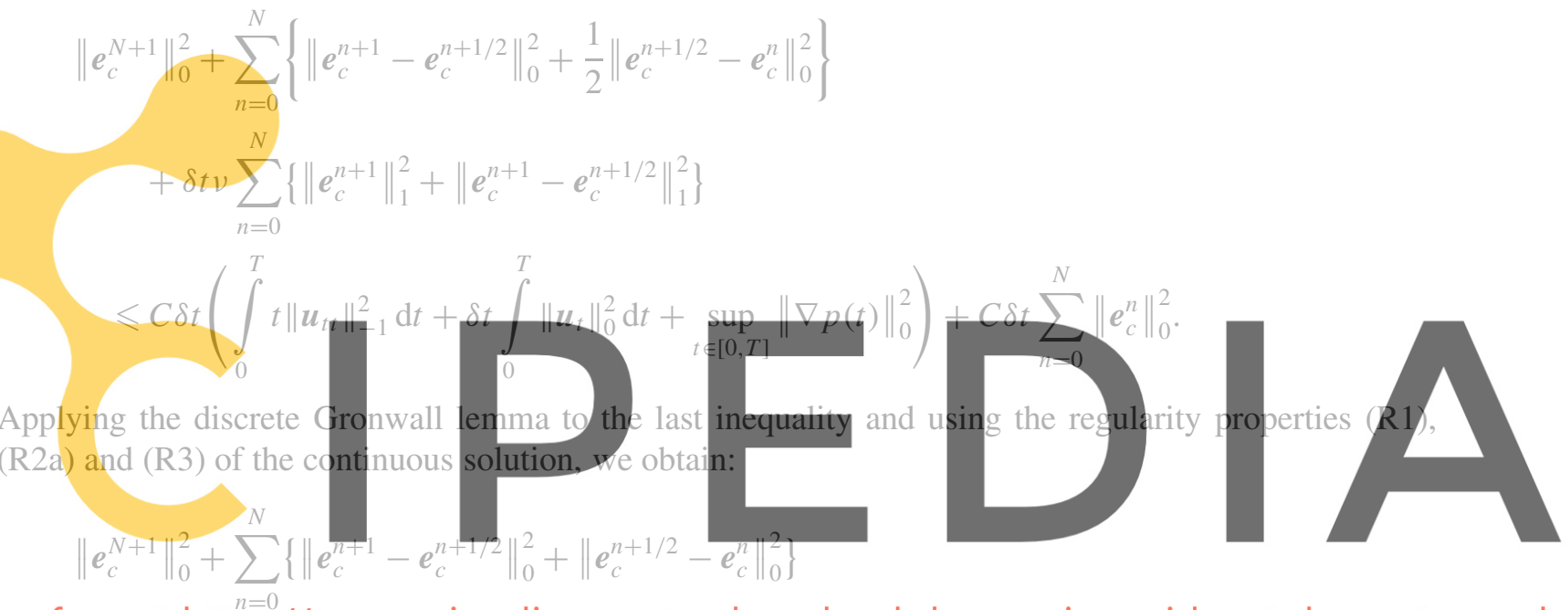

egister for free at https//www.scipedia.com to download the version without the watermark

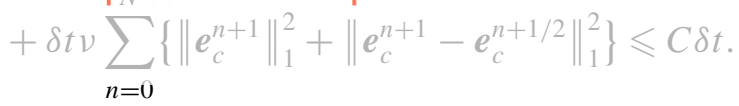

Finally, the bounds for $\boldsymbol{u}^{n+1 / 2}$ follow from (22) and the triangle inequality, so that (14) is proved.

Remark 2. Lemma 1 shows, in particular, that the method provides uniformly stable velocities in $\mathbf{H}_{0}^{1}(\Omega)$, that is to say, that there exists a constant $C>0$ independent of the time step $\delta t$ such that for all $n=0, \ldots,[T / \delta t]-1$ :

$$
\left\|\boldsymbol{u}^{n+1}\right\|_{1} \leqslant C, \quad\left\|\boldsymbol{u}^{n+1 / 2}\right\|_{1} \leqslant C,
$$

since $\left\|\boldsymbol{e}_{c}^{n+1}\right\|_{1} \leqslant C,\left\|\boldsymbol{e}_{c}^{n+1 / 2}\right\|_{1} \leqslant C$ and $\boldsymbol{u} \in L^{\infty}\left(0, T ; \mathbf{H}_{0}^{1}(\Omega)\right)$. Moreover, we also have:

$$
\left\|\boldsymbol{e}_{c}^{n+1}\right\|_{0} \leqslant C \delta t^{1 / 2}, \quad\left\|\boldsymbol{e}_{c}^{n+1 / 2}\right\|_{0} \leqslant C \delta t^{1 / 2} .
$$

We will use these bounds later on.

Next we give a first order error estimate for both $\boldsymbol{u}^{n+1 / 2}$ and $\boldsymbol{u}^{n+1}$ in the norm of $l^{2}\left(\mathbf{L}^{2}(\Omega)\right)$, which is what was proved for the standard projection method in [20] when applied to the (linear) Stokes problem, that is, when dropping the convective term in (1): 
Theorem 3. Assume (R1), (R2a), (R3) and (R4) hold; then, for $N=0, \ldots,[T / \delta t]-1$ and for small enough $\delta t$ :

$$
\left\|\boldsymbol{e}_{c}^{N+1}\right\|_{V^{\prime}}^{2}+\delta t \sum_{n=0}^{N}\left(\left\|\boldsymbol{e}_{c}^{n+1}\right\|_{0}^{2}+\left\|\boldsymbol{e}_{c}^{n+1 / 2}\right\|_{0}^{2}\right) \leqslant C \delta t^{2},
$$

that is, $\boldsymbol{u}^{n+1}$ converges to $\boldsymbol{u}\left(t_{n+1}\right)$ in $l^{\infty}\left(V^{\prime}\right) \cap l^{2}\left(\mathbf{L}^{2}(\Omega)\right)$ and $\boldsymbol{u}^{n+1 / 2}$ in $l^{2}\left(\mathbf{L}^{2}(\Omega)\right)$ with order $\delta t$.

Proof. By adding (9) and (11), we get:

$$
\frac{\boldsymbol{u}^{n+1}-\boldsymbol{u}^{n}}{\delta t}-v \Delta \boldsymbol{u}^{n+1}+\left(\boldsymbol{u}^{n} \cdot \nabla\right) \boldsymbol{u}^{n+1 / 2}+\nabla p^{n+1}=\boldsymbol{f}\left(t_{n+1}\right)
$$

Calling $r_{c}^{n+1}=p\left(t_{n+1}\right)-p^{n+1}$ the pressure error and subtracting (26) from (15), we have:

$$
\frac{1}{\delta t}\left(\boldsymbol{e}_{c}^{n+1}-\boldsymbol{e}_{c}^{n}\right)-v \Delta\left(\boldsymbol{e}_{c}^{n+1}\right)+\nabla r_{c}^{n+1}=\left(\boldsymbol{u}^{n} \cdot \nabla\right) \boldsymbol{u}^{n+1 / 2}-\left(\boldsymbol{u}\left(t_{n+1}\right) \cdot \nabla\right) \boldsymbol{u}\left(t_{n+1}\right)+\mathbf{R}^{n}
$$

We take the inner product of (27) with $2 \delta t A^{-1} \boldsymbol{e}_{c}^{n+1}$, as in [20], and use the self-adjointness of $A^{-1}$ to get:

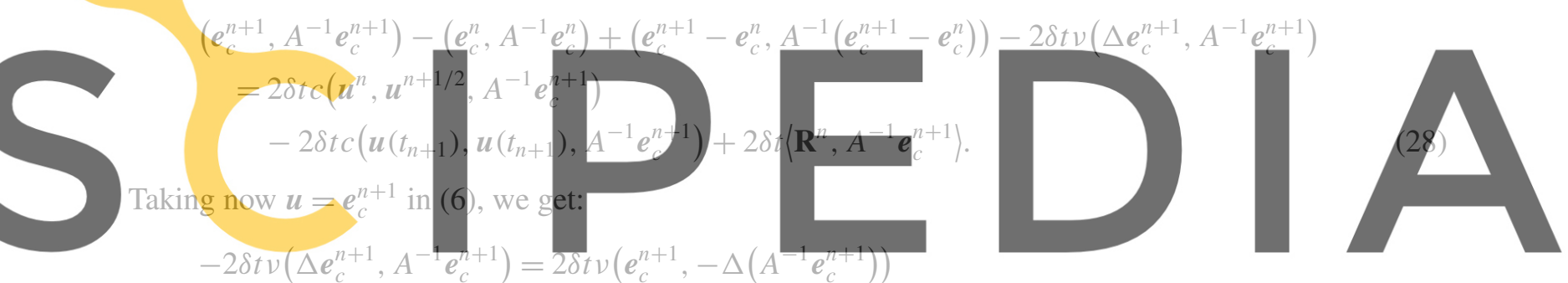

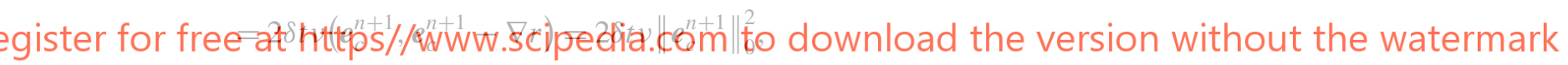

since $\nabla \cdot e_{c}^{n+1}=0$. The RHS terms in (28) are bounded as follows. For the Taylor residual term, we have:

$$
\begin{gathered}
2 \delta t\left\langle\mathbf{R}^{n}, A^{-1} \boldsymbol{e}_{c}^{n+1}\right\rangle \leqslant 2 \delta t\left\|\mathbf{R}^{n}\right\|_{V^{\prime}}\left\|A^{-1} \boldsymbol{e}_{c}^{n+1}\right\|_{1} \leqslant 2 \delta t\left\|\mathbf{R}^{n}\right\|_{V^{\prime}}\left\|\boldsymbol{e}_{c}^{n+1}\right\|_{V^{\prime}} \\
\leqslant \delta t\left\|\boldsymbol{e}_{c}^{n+1}\right\|_{V^{\prime}}^{2}+C \delta t\left\|\mathbf{R}^{n}\right\|_{V^{\prime}}^{2} \leqslant \delta t\left\|\boldsymbol{e}_{c}^{n+1}\right\|_{V^{\prime}}^{2}+C \delta t^{2} \int_{t_{n}}^{t_{n+1}}\left\|\boldsymbol{u}_{t t}\right\|_{V^{\prime}}^{2} \mathrm{~d} t .
\end{gathered}
$$

For the nonlinear terms, we use the splitting (17) again and bound the corresponding three terms as follows:

$$
\begin{aligned}
& -2 \delta t c\left(\boldsymbol{u}\left(t_{n+1}\right), \boldsymbol{e}_{c}^{n+1 / 2}, A^{-1} \boldsymbol{e}_{c}^{n+1}\right) \leqslant C \delta t\left\|\boldsymbol{u}\left(t_{n+1}\right)\right\|_{2}\left\|A^{-1} \boldsymbol{e}_{c}^{n+1}\right\|_{1}\left\|\boldsymbol{e}_{c}^{n+1 / 2}\right\|_{0} \\
& \leqslant C \delta t\left\|\boldsymbol{e}_{c}^{n+1}\right\|_{V^{\prime}}^{2}+\frac{\delta t v}{4}\left\|\boldsymbol{e}_{c}^{n+1 / 2}\right\|_{0}^{2} \\
& =C \delta t\left\|\boldsymbol{e}_{c}^{n+1}\right\|_{V^{\prime}}^{2}+\frac{\delta t v}{4}\left\{\left\|\boldsymbol{e}_{c}^{n+1}\right\|_{0}^{2}+\left\|\boldsymbol{e}_{c}^{n+1}-\boldsymbol{e}_{c}^{n+1 / 2}\right\|_{0}^{2}\right. \\
& \left.\quad+\delta t v\left\|\boldsymbol{e}_{c}^{n+1}\right\|_{1}^{2}+\delta t v\left\|\boldsymbol{e}_{c}^{n+1}-\boldsymbol{e}_{c}^{n+1 / 2}\right\|_{1}^{2}-\delta t v\left\|\boldsymbol{e}_{c}^{n+1 / 2}\right\|_{1}^{2}\right\},
\end{aligned}
$$

where we have used (5) and (21); 


$$
\begin{aligned}
& 2 \delta t c\left(\boldsymbol{u}\left(t_{n}\right)-\boldsymbol{u}\left(t_{n+1}\right), \boldsymbol{u}^{n+1 / 2}, A^{-1} \boldsymbol{e}_{c}^{n+1}\right) \\
& \quad \leqslant C \delta t\left\|\boldsymbol{u}\left(t_{n}\right)-\boldsymbol{u}\left(t_{n+1}\right)\right\|_{0}\left\|\boldsymbol{u}^{n+1 / 2}\right\|_{1}\left\|A^{-1} \boldsymbol{e}_{c}^{n+1}\right\|_{2} \\
& \quad \leqslant C \delta t\left\|\int_{t_{n}}^{t_{n+1}} \boldsymbol{u}_{t} \mathrm{~d} t\right\|_{0}^{t_{n+1}}\left\|\boldsymbol{e}_{c}^{n+1}\right\|_{0} \leqslant C \delta t^{2} \int_{t_{n}}\left\|\boldsymbol{u}_{t}\right\|_{0}^{2} \mathrm{~d} t+\frac{\delta t v}{4}\left\|\boldsymbol{e}_{c}^{n+1}\right\|_{0}^{2},
\end{aligned}
$$

where we have used (23); and:

$$
\begin{aligned}
& -2 \delta t c\left(\boldsymbol{e}_{c}^{n}, \boldsymbol{u}^{n+1 / 2}, A^{-1} \boldsymbol{e}_{c}^{n+1}\right) \\
& \quad=2 \delta t c\left(\boldsymbol{e}_{c}^{n}, A^{-1} \boldsymbol{e}_{c}^{n+1}, \boldsymbol{u}\left(t_{n+1}\right)\right)-2 \delta t c\left(\boldsymbol{e}_{c}^{n}, A^{-1} \boldsymbol{e}_{c}^{n+1}, \boldsymbol{e}_{c}^{n+1 / 2}\right)=\mathrm{T}_{1}+\mathrm{T}_{2},
\end{aligned}
$$

So that:

$$
\begin{aligned}
\mathrm{T}_{1} & \leqslant C \delta t\left\|\boldsymbol{e}_{c}^{n}\right\|_{0}\left\|A^{-1} \boldsymbol{e}_{c}^{n+1}\right\|_{1}\left\|\boldsymbol{u}\left(t_{n+1}\right)\right\|_{2} \\
& \leqslant C \delta t\left\|\boldsymbol{e}_{c}^{n}\right\|_{0}\left\|\boldsymbol{e}_{c}^{n+1}\right\|_{V^{\prime}} \leqslant C \delta t\left(\left\|\boldsymbol{e}_{c}^{n+1}\right\|_{0}+\left\|\boldsymbol{e}_{c}^{n+1}-\boldsymbol{e}_{c}^{n+1 / 2}\right\|_{0}+\left\|\boldsymbol{e}_{c}^{n+1 / 2}-\boldsymbol{e}_{c}^{n}\right\|_{0}\right)\left\|\boldsymbol{e}_{c}^{n+1}\right\|_{V^{\prime}} \\
& \leqslant \frac{\delta t v}{4}\left\|\boldsymbol{e}_{c}^{n+1}\right\|_{0}^{2}+C \delta t\left(\left\|\boldsymbol{e}_{c}^{n+1}-\boldsymbol{e}_{c}^{n+1 / 2}\right\|_{0}^{2}+\left\|\boldsymbol{e}_{c}^{n+1 / 2}-\boldsymbol{e}_{c}^{n}\right\|_{0}^{2}\right)+C \delta t\left\|\boldsymbol{e}_{c}^{n+1}\right\|_{V^{\prime}}^{2},
\end{aligned}
$$

due to (R1); and finally:

$$
\begin{aligned}
\mathrm{T}_{2} & \leqslant C \delta t\left\|\boldsymbol{e}_{c}^{n}\right\|_{0}\left\|A^{-1} \boldsymbol{e}_{c}^{n+1}\right\|_{2}\left\|\boldsymbol{e}_{c}^{n+1 / 2}\right\|_{1} \leqslant C \delta t\left\|\boldsymbol{e}_{c}^{n}\right\|_{0}\left\|\boldsymbol{e}_{c}^{n+1}\right\|_{0}\left\|\boldsymbol{e}_{c}^{n+1 / 2}\right\|_{1} \\
& \leqslant C \delta t^{3 / 2}\left\|\boldsymbol{e}_{c}^{n+1}\right\|_{0}\left\|\boldsymbol{e}_{c}^{n+1 / 2}\right\|_{1} \leqslant \frac{\delta t v}{4}\left\|\boldsymbol{e}_{c}^{n+1}\right\|_{0}^{2}+C \delta t^{2}\left\|\boldsymbol{e}_{c}^{n+1 / 2}\right\|_{1}^{2},
\end{aligned}
$$

where we have used (24). Adding up (28) for $n=0, \ldots, N$, and using all these inequalities, we get:

$$
\begin{aligned}
& \left(\boldsymbol{e}_{c}^{N+1}, A^{-1} \boldsymbol{e}_{c}^{N+1}\right)+\sum_{n=0}^{N}\left(\boldsymbol{e}_{c}^{n+1}-\boldsymbol{e}_{c}^{n}, A^{-1}\left(\boldsymbol{e}_{c}^{n+1}-\boldsymbol{e}_{c}^{n}\right)\right)+\delta t v \sum_{n=0}^{N}\left\|\boldsymbol{e}_{c}^{n+1}\right\|_{0}^{2} \\
& \leqslant C \delta t^{2} \int_{0}^{T}\left\|\boldsymbol{u}_{t}\right\|_{V^{\prime}}^{2} \mathrm{~d} t+C \delta t^{2} \int_{0}^{T}\left\|\boldsymbol{u}_{t}\right\|_{0}^{2} \mathrm{~d} t+C \delta t \sum_{n=0}^{N}\left\|\boldsymbol{e}_{c}^{n+1}\right\|_{V^{\prime}}^{2}+C \delta t^{2} \sum_{n=0}^{N}\left\|\boldsymbol{e}_{c}^{n+1}\right\|_{1}^{2} \\
& \quad+C \delta t \sum_{n=0}^{N}\left\{\left\|\boldsymbol{e}_{c}^{n+1}-\boldsymbol{e}_{c}^{n+1 / 2}\right\|_{0}^{2}+\left\|\boldsymbol{e}_{c}^{n+1 / 2}-\boldsymbol{e}_{c}^{n}\right\|_{0}^{2}\right\}+C \delta t^{2} \sum_{n=0}^{N}\left\|\boldsymbol{e}_{c}^{n+1}-\boldsymbol{e}_{c}^{n+1 / 2}\right\|_{1}^{2} \\
& \quad+C \delta t^{2} \sum_{n=0}^{N}\left\|\boldsymbol{e}_{c}^{n+1 / 2}\right\|_{1}^{2} .
\end{aligned}
$$

Using now (8), the regularity properties (R2a) and (R4) of the continuous solution and the estimates of Lemma 1, we get:

$$
\left\|\boldsymbol{e}_{c}^{N+1}\right\|_{V^{\prime}}^{2}+\sum_{n=0}^{N}\left\|\boldsymbol{e}_{c}^{n+1}-\boldsymbol{e}_{c}^{n}\right\|_{V^{\prime}}^{2}+\delta t v \sum_{n=0}^{N}\left\|\boldsymbol{e}_{c}^{n+1}\right\|_{0}^{2} \leqslant C \delta t^{2}+C \delta t \sum_{n=0}^{N}\left\|\boldsymbol{e}_{c}^{n+1}\right\|_{V^{\prime}}^{2} .
$$

For sufficiently small $\delta t$, we can apply the discrete Gronwall lemma to the last inequality, and we get:

$$
\left\|\boldsymbol{e}_{c}^{N+1}\right\|_{V^{\prime}}^{2}+\sum_{n=0}^{N}\left\|\boldsymbol{e}_{c}^{n+1}-\boldsymbol{e}_{c}^{n}\right\|_{V^{\prime}}^{2}+\delta t v \sum_{n=0}^{N}\left\|\boldsymbol{e}_{c}^{n+1}\right\|_{0}^{2} \leqslant C \delta t^{2},
$$


and the estimate for $\boldsymbol{u}^{n+1}$ is proved. For $\boldsymbol{u}^{n+1 / 2}$, we have:

$$
\delta t v \sum_{n=0}^{N}\left\|\boldsymbol{e}_{c}^{n+1 / 2}\right\|_{0}^{2} \leqslant 2 \delta t v \sum_{n=0}^{N}\left(\left\|\boldsymbol{e}_{c}^{n+1}\right\|_{0}^{2}+\left\|\boldsymbol{e}_{c}^{n+1}-\boldsymbol{e}_{c}^{n+1 / 2}\right\|_{0}^{2}\right) \leqslant C \delta t^{2},
$$

due to (29) and Lemma 1 , so that (25) is proved.

The estimates of Theorem 3 allow us to obtain now enhanced stability properties of the semidiscrete solution.

Theorem 4. Assume that (R1), (R2a), (R3) and (R4) hold; assume also that $f \in L^{\infty}(0, T ; H)$ and that the domain $\Omega$ is of class $\mathcal{C}^{2}$ (or is a convex polygon or polyhedron); then, for $N=0, \ldots,[T / \delta t]-1$, and for small enough $\delta t$ :

$$
\delta t \sum_{n=0}^{N}\left\{\left\|\boldsymbol{u}^{n+1}\right\|_{2}^{2}+\left\|\boldsymbol{u}^{n+1 / 2}\right\|_{2}^{2}\right\} \leqslant C, \quad \delta t \sum_{n=0}^{N}\left\|p^{n+1}\right\|_{1}^{2} \leqslant C,
$$

that is, $\boldsymbol{u}^{n+1}$ and $\boldsymbol{u}^{n+1 / 2}$ are uniformly bounded in $l^{2}\left(\mathbf{H}^{2}(\Omega)\right)$ and $p^{n+1}$ is uniformly bounded in $l^{2}\left(\mathbf{H}^{1}(\Omega)\right)$.

Proof. We use a similar argument to that of [24, Theorem III.3.8]. We rewrite (9) as:

$$
-v \Delta \boldsymbol{u}^{n+1 / 2}=\boldsymbol{f}\left(t_{n+1}\right)-\frac{1}{\delta t}\left(\boldsymbol{u}^{n+1 / 2}-\boldsymbol{u}^{n}\right)-\left(\boldsymbol{u}^{n} \cdot \nabla\right) \boldsymbol{u}^{n+1 / 2} .
$$

Then:

$$
\begin{aligned}
\delta t & \sum_{n=0}^{N}\left\|\frac{1}{\delta t}\left(\boldsymbol{u}^{n+1 / 2}-\boldsymbol{u}^{n}\right)\right\|_{0}^{2} \\
& \leqslant \frac{C}{\delta t} \sum_{n=0}^{N}\left\{\left\|\boldsymbol{u}^{n+1 / 2}-\boldsymbol{u}\left(t_{n+1}\right)\right\|_{0}^{2}+\left\|\boldsymbol{u}\left(t_{n+1}\right)-\boldsymbol{u}\left(t_{n}\right)\right\|_{0}^{2}+\left\|\boldsymbol{u}\left(t_{n}\right)-\boldsymbol{u}^{n}\right\|_{0}^{2}\right\} \\
& \leqslant \frac{C}{\delta t} \sum_{n=0}^{N}\left\{\left\|\boldsymbol{e}_{c}^{n+1 / 2}\right\|_{0}^{2}+\delta t \int_{t_{n}}^{t_{n+1}}\left\|\boldsymbol{u}_{t}\right\|_{0}^{2} \mathrm{~d} t+\left\|\boldsymbol{e}_{c}^{n}\right\|_{0}^{2}\right\} \leqslant C,
\end{aligned}
$$

due to Theorem 3 and assumption (R2a). Moreover:

$$
\left\|\left(\boldsymbol{u}^{n} \cdot \nabla\right) \boldsymbol{u}^{n+1 / 2}\right\|_{\mathbf{L}^{3 / 2}(\Omega)}=\sup _{\boldsymbol{w} \in \mathbf{L}^{3}(\Omega)} \frac{\left(\left(\boldsymbol{u}^{n} \cdot \nabla\right) \boldsymbol{u}^{n+1 / 2}, \boldsymbol{w}\right)}{\|\boldsymbol{w}\|_{\mathbf{L}^{3}(\Omega)}} \leqslant C\left\|\boldsymbol{u}^{n}\right\|_{1}\left\|\boldsymbol{u}^{n+1 / 2}\right\|_{1} \leqslant C,
$$

due to the continuity properties of the trilinear form $c$ and Remark 2; from (30), we can now deduce that $\Delta \boldsymbol{u}^{n+1 / 2}$ is bounded in $l^{2}\left(\mathbf{L}^{3 / 2}(\Omega)\right)$. Next, we rewrite (11) as:

$$
\begin{aligned}
& -v \Delta \boldsymbol{u}^{n+1}+\nabla p^{n+1}=-v \Delta \boldsymbol{u}^{n+1 / 2}-\frac{1}{\delta t}\left(\boldsymbol{u}^{n+1}-\boldsymbol{u}^{n+1 / 2}\right), \\
& \nabla \cdot \boldsymbol{u}^{n+1}=0 \\
& \left.\boldsymbol{u}^{n+1}\right|_{\partial \Omega}=0 .
\end{aligned}
$$


The term $\frac{1}{\delta t}\left(\boldsymbol{u}^{n+1}-\boldsymbol{u}^{n+1 / 2}\right)$ can be easily bounded in $l^{2}\left(\mathbf{L}^{2}(\Omega)\right)$ as before, so that, using the regularity of solutions of the Stokes problem (31) on regular domains, we can assure that $\boldsymbol{u}^{n+1}$ is bounded in $l^{2}\left(\mathbf{W}^{2,3 / 2}(\Omega)\right)$ and $p^{n+1}$ is bounded in $l^{2}\left(W^{1,3 / 2}(\Omega)\right)$. Due to Sobolev's compactness theorem, we then have that $\boldsymbol{u}^{n+1}$ is bounded in $l^{2}\left(\mathbf{L}^{8}(\Omega)\right)$ both when $d=2$ and 3. Furthermore:

$$
\left\|\left(\boldsymbol{u}^{n} \cdot \nabla\right) \boldsymbol{u}^{n+1 / 2}\right\|_{\mathbf{L}^{8 / 5}(\Omega)}=\sup _{\boldsymbol{w} \in \mathbf{L}^{8 / 3}(\Omega)} \frac{\left(\left(\boldsymbol{u}^{n} \cdot \nabla\right) \boldsymbol{u}^{n+1 / 2}, \boldsymbol{w}\right)}{\|\boldsymbol{w}\|_{\mathbf{L}^{8 / 3}(\Omega)}} \leqslant C\left\|\boldsymbol{u}^{n}\right\|_{\mathbf{L}^{8}(\Omega)}\left\|\boldsymbol{u}^{n+1 / 2}\right\|_{1},
$$

according to the last property of the form $c$ on page 5 , which ensures that $\left(\boldsymbol{u}^{n} \cdot \nabla\right) \boldsymbol{u}^{n+1 / 2}$ is bounded in $l^{2}\left(\mathbf{L}^{8 / 5}(\Omega)\right)$. Returning to (30), we improve the regularity of $\Delta \boldsymbol{u}^{n+1 / 2}$ to $l^{2}\left(\mathbf{L}^{8 / 5}(\Omega)\right)$, and then that of $\boldsymbol{u}^{n+1}$ to $l^{2}\left(\mathbf{W}^{2,8 / 5}(\Omega)\right)$ and $p^{n+1}$ to $l^{2}\left(W^{1,8 / 5}(\Omega)\right)$, as solutions of the Stokes problem (31). Sobolev's theorem ensures now that $\boldsymbol{u}^{n+1}$ is bounded in $l^{2}\left(\mathbf{L}^{\infty}(\Omega)\right)$. This fact, together with Remark 2, implies that $\left(\boldsymbol{u}^{n} \cdot \nabla\right) \boldsymbol{u}^{n+1 / 2}$ is bounded in $l^{2}\left(\mathbf{L}^{2}(\Omega)\right)$, which, returning to (30) once more, provides a bound for $\Delta \boldsymbol{u}^{n+1 / 2}$ also in $l^{2}\left(\mathbf{L}^{2}(\Omega)\right)$, which is sufficient to bound $\boldsymbol{u}^{n+1 / 2}$ in $l^{2}\left(\mathbf{H}^{2}(\Omega)\right)$ when $\Omega$ is regular enough (see [10]). Finally, the bounds for $\boldsymbol{u}^{n+1}$ and $p^{n+1}$ follow again from the regularity of the Stokes problem.

The error estimates of Theorem 3 can be improved to first order in the norms of $l^{\infty}\left(\mathbf{L}^{2}(\Omega)\right)$ and $l^{2}\left(\mathbf{H}_{0}^{1}(\Omega)\right)$ for the end-of-step velocities $\boldsymbol{u}^{n+1}$ assuming some slightly stronger regularity on the continuous solution, namely, (R2b) rather than (R2a). Estimates in these norms were also obtained in [12] for the intermediate velocities of a fully discrete, incremental version of the fractional step projection method, assuming a finite element spatial discretization satisfying the discrete inf-sup condition and under much stronger regularity assumptions on the continuous solution:

Theorem 5. Assume that (R1), (R2b), (R3) and (R4) hold; then, for $N=0, \ldots,[T / \delta t]-1$, and for small enough $\delta t$ :

$$
\left\|\boldsymbol{e}_{c}^{N+1}\right\|_{0}^{2}+\delta t v \sum_{n=0}^{N}\left\|\boldsymbol{e}_{c}^{n+1}\right\|_{1}^{2} \leqslant C \delta t^{2}
$$

that is, $\boldsymbol{u}^{n+1}$ converges to $\boldsymbol{u}\left(t_{n+1}\right)$ in $l^{\infty}\left(\mathbf{L}^{2}(\Omega)\right) \cap l^{2}\left(\mathbf{H}_{0}^{1}(\Omega)\right)$ with order $\delta t$.

Proof. Unlike in the standard projection method, we can take the inner product of (27) with $2 \delta t \boldsymbol{e}_{c}^{n+1}$, since in our case $\boldsymbol{e}_{c}^{n+1} \in V$, to get:

$$
\begin{aligned}
& \left\|\boldsymbol{e}_{c}^{n+1}\right\|_{0}^{2}-\left\|\boldsymbol{e}_{c}^{n}\right\|_{0}^{2}+\left\|\boldsymbol{e}_{c}^{n+1}-\boldsymbol{e}_{c}^{n}\right\|_{0}^{2}+2 \delta t v\left\|\boldsymbol{e}_{c}^{n+1}\right\|_{1}^{2} \\
& \quad=2 \delta t c\left(\boldsymbol{u}^{n}, \boldsymbol{u}^{n+1 / 2}, \boldsymbol{e}_{c}^{n+1}\right)-2 \delta t c\left(\boldsymbol{u}\left(t_{n+1}\right), \boldsymbol{u}\left(t_{n+1}\right), \boldsymbol{e}_{c}^{n+1}\right)+2 \delta t\left\langle\mathbf{R}^{n}, \boldsymbol{e}_{c}^{n+1}\right\rangle .
\end{aligned}
$$

The RHS terms in (33) are bounded as follows. For the Taylor residual term, we have:

$$
2 \delta t\left\langle\mathbf{R}^{n}, \boldsymbol{e}_{c}^{n+1}\right\rangle \leqslant 2 \delta t\left\|\mathbf{R}^{n}\right\|_{V^{\prime}}\left\|\boldsymbol{e}_{c}^{n+1}\right\|_{1} \leqslant \frac{\delta t v}{5}\left\|\boldsymbol{e}_{c}^{n+1}\right\|_{1}^{2}+C \delta t^{2} \int_{t_{n}}^{t_{n+1}}\left\|\boldsymbol{u}_{t t}\right\|_{V^{\prime}}^{2} \mathrm{~d} t .
$$

For the nonlinear terms, we use again the splitting (17) and bound the corresponding terms as: 


$$
\begin{aligned}
& -2 \delta t c\left(\boldsymbol{u}\left(t_{n+1}\right), \boldsymbol{e}_{c}^{n+1 / 2}, \boldsymbol{e}_{c}^{n+1}\right) \\
& \quad \leqslant C \delta t\left\|\boldsymbol{u}\left(t_{n+1}\right)\right\|_{2}\left\|\boldsymbol{e}_{c}^{n+1}\right\|_{1}\left\|\boldsymbol{e}_{c}^{n+1 / 2}\right\|_{0} \leqslant \frac{\delta t v}{5}\left\|\boldsymbol{e}_{c}^{n+1}\right\|_{1}^{2}+C \delta t\left\|\boldsymbol{e}_{c}^{n+1 / 2}\right\|_{0}^{2}, \\
& 2 \delta t c\left(\boldsymbol{u}\left(t_{n}\right)-\boldsymbol{u}\left(t_{n+1}\right), \boldsymbol{u}^{n+1 / 2}, \boldsymbol{e}_{c}^{n+1}\right) \\
& \quad \leqslant C \delta t\left\|\boldsymbol{u}\left(t_{n}\right)-\boldsymbol{u}\left(t_{n+1}\right)\right\|_{1}\left\|\boldsymbol{u}^{n+1 / 2}\right\|_{1}\left\|\boldsymbol{e}_{c}^{n+1}\right\|_{1} \leqslant C \delta t^{2} \int_{t_{n}}^{t_{n+1}}\left\|\boldsymbol{u}_{t}\right\|_{1}^{2} \mathrm{~d} t+\frac{\delta t v}{5}\left\|\boldsymbol{e}_{c}^{n+1}\right\|_{1}^{2}, \\
& -2 \delta t c\left(\boldsymbol{e}_{c}^{n}, \boldsymbol{u}^{n+1 / 2}, \boldsymbol{e}_{c}^{n+1}\right)=2 \delta t c\left(\boldsymbol{e}_{c}^{n}, \boldsymbol{e}_{c}^{n+1 / 2}, \boldsymbol{e}_{c}^{n+1}\right)-2 \delta t c\left(\boldsymbol{e}_{c}^{n}, \boldsymbol{u}\left(t_{n+1}\right), \boldsymbol{e}_{c}^{n+1}\right)=\mathrm{T}_{1}+\mathrm{T}_{2},
\end{aligned}
$$

so that:

$$
\begin{aligned}
\mathrm{T}_{1} & \leqslant C \delta t\left\|\boldsymbol{e}_{c}^{n}\right\|_{1}\left\|\boldsymbol{e}_{c}^{n+1}\right\|_{1}\left\|\boldsymbol{e}_{c}^{n+1 / 2}\right\|_{0}^{1 / 2}\left\|\boldsymbol{e}_{c}^{n+1 / 2}\right\|_{1}^{1 / 2} \\
& \leqslant C \delta t\left\|\boldsymbol{e}_{c}^{n}\right\|_{1}\left\|\boldsymbol{e}_{c}^{n+1}\right\|_{1}\left\|\boldsymbol{e}_{c}^{n+1 / 2}\right\|_{0}^{1 / 2} \leqslant C \delta t^{5 / 4}\left\|\boldsymbol{e}_{c}^{n}\right\|_{1}\left\|\boldsymbol{e}_{c}^{n+1}\right\|_{1} \leqslant C \delta t^{3 / 2} v\left\|\boldsymbol{e}_{c}^{n}\right\|_{1}^{2}+\frac{\delta t v}{5}\left\|\boldsymbol{e}_{c}^{n+1}\right\|_{1}^{2}, \\
\mathrm{~T}_{2} & \leqslant C \delta t\left\|\boldsymbol{e}_{c}^{n}\right\|_{0}\left\|\boldsymbol{u}\left(t_{n+1}\right)\right\|_{2}\left\|\boldsymbol{e}_{c}^{n+1}\right\|_{1} \leqslant C \delta t\left\|\boldsymbol{e}_{c}^{n}\right\|_{0}^{2}+\frac{\delta t v}{5}\left\|\boldsymbol{e}_{c}^{n+1}\right\|_{1}^{2},
\end{aligned}
$$

where we have used (24) and the continuity properties of the trilinear form $c$. Adding up (33) for $n=0, \ldots, N$ and taking into account (21) and the previous inequalities, we get:

$$
\begin{aligned}
&\left\|\boldsymbol{e}_{c}^{N+1}\right\|_{0}^{2}+\sum_{n=0}^{N}\left\|\boldsymbol{e}_{c}^{n+1}-\boldsymbol{e}_{c}^{n}\right\|_{0}^{2}+\delta t v \sum_{n=0}^{N}\left\|\boldsymbol{e}_{c}^{n+1}\right\|_{1}^{2}+C \delta t^{2} v \sum_{n=0}^{N}\left\|\boldsymbol{e}_{c}^{n+1 / 2}\right\|_{1}^{2} \\
& \leqslant C \delta t^{2} \int_{0}^{T}\left\|\boldsymbol{u}_{t}\right\|_{V^{\prime}}^{2} \mathrm{~d} t+C \delta t^{2} \int_{0}^{T}\left\|\boldsymbol{u}_{t}\right\|_{1}^{2} \mathrm{~d} t+C \delta t \sum_{n=0}^{N}\left\|\boldsymbol{e}_{c}^{n+1}\right\|_{0}^{2}+C \delta t \sum_{n=0}^{N}\left\|\boldsymbol{e}_{c}^{n+1}-\boldsymbol{e}_{c}^{n+1 / 2}\right\|_{0}^{2} \\
&+C \delta t^{2} \sum_{n=0}^{N}\left\{\left\|\boldsymbol{e}_{c}^{n+1}\right\|_{1}^{2}+\left\|\boldsymbol{e}_{c}^{n+1}-\boldsymbol{e}_{c}^{n+1 / 2}\right\|_{1}^{2}\right\}+C \delta t^{3 / 2} v \sum_{n=0}^{N}\left\|\boldsymbol{e}_{c}^{n}\right\|_{1}^{2} .
\end{aligned}
$$

Using the regularity properties of the solution (R2b) and (R4) and the estimates of Lemma 1, we get:

$$
\begin{aligned}
& \left\|\boldsymbol{e}_{c}^{N+1}\right\|_{0}^{2}+\sum_{n=0}^{N}\left\|\boldsymbol{e}_{c}^{n+1}-\boldsymbol{e}_{c}^{n}\right\|_{0}^{2}+\delta t v \sum_{n=0}^{N}\left\|\boldsymbol{e}_{c}^{n+1}\right\|_{1}^{2}+C \delta t^{2} v \sum_{n=0}^{N}\left\|\boldsymbol{e}_{c}^{n+1 / 2}\right\|_{1}^{2} \\
& \leqslant C \delta t^{2}+C \delta t \sum_{n=0}^{N}\left\|\boldsymbol{e}_{c}^{n+1}\right\|_{0}^{2}+C \delta t^{3 / 2} v \sum_{n=0}^{N}\left\|\boldsymbol{e}_{c}^{n}\right\|_{1}^{2} .
\end{aligned}
$$

For sufficiently small $\delta t$, we can apply the discrete Gronwall lemma to the last inequality and take the last term to the left side, to get:

$$
\left\|\boldsymbol{e}_{c}^{N+1}\right\|_{0}^{2}+\sum_{n=0}^{N}\left\|\boldsymbol{e}_{c}^{n+1}-\boldsymbol{e}_{c}^{n}\right\|_{0}^{2}+\delta t v \sum_{n=0}^{N}\left\|\boldsymbol{e}_{c}^{n+1}\right\|_{1}^{2} \leqslant C \delta t^{2},
$$

and (25) is proved. 


\subsection{Error estimates for the semidiscrete pressure}

As a side product of the estimates of Theorem 5, we obtain order 1/2 error estimates for the pressure approximation in $l^{2}\left(L_{0}^{2}(\Omega)\right)$, which is what one can expect for the present scheme. We first recall a technical result, similar to that of [21, Lemma A1]. In Theorem 5 we have proved, in particular, that:

$$
\sum_{n=0}^{N}\left\|\boldsymbol{e}_{c}^{n+1}-\boldsymbol{e}_{c}^{n}\right\|_{0}^{2} \leqslant C \delta t^{2} .
$$

This implies that:

$$
\sum_{n=0}^{N}\left\|\boldsymbol{e}_{c}^{n+1}-\boldsymbol{e}_{c}^{n}\right\|_{-1}^{2} \leqslant C \delta t^{2},
$$

since for all $\boldsymbol{v} \in \mathbf{L}^{2}(\Omega),\|\boldsymbol{v}\|_{-1} \leqslant\|\boldsymbol{v}\|_{0}$. This is what we actually use to prove the following error estimate for the pressure:

Theorem 6. Assume that (R1), (R2b), (R3) and (R4) hold; then, for $N=0, \ldots,[T / \delta t]-1$ and for small enough $\delta t$ :

$$
\delta t \sum_{n=0}^{N}\left\|p\left(t_{n+1}\right)-p^{n+1}\right\|_{L_{0}^{2}(\Omega)}^{2} \leqslant C \delta t,
$$

that is, $p^{n+1}$ converges to $p\left(t_{n+1}\right)$ in $l^{2}\left(L_{0}^{2}(\Omega)\right)$ with order $\delta t^{1 / 2}$.

Proof. We rewrite (27) as:

$$
-\nabla r_{c}^{n+1}=\frac{1}{\delta t}\left(\boldsymbol{e}_{c}^{n+1}-\boldsymbol{e}_{c}^{n}\right)-v \Delta\left(\boldsymbol{e}_{c}^{n+1}\right)-\mathbf{R}^{n}-\left(\boldsymbol{u}^{n} \cdot \nabla\right) \boldsymbol{u}^{n+1 / 2}+\left(\boldsymbol{u}\left(t_{n+1}\right) \cdot \nabla\right) \boldsymbol{u}\left(t_{n+1}\right) .
$$

Using the continuous LBB condition:

$$
\left\|r_{c}^{n+1}\right\|_{L_{0}^{2}(\Omega)} \leqslant C \sup _{\boldsymbol{v} \in \mathbf{H}_{0}^{1}(\Omega)} \frac{\left(\nabla r_{c}^{n+1}, \boldsymbol{v}\right)}{\|\boldsymbol{v}\|_{1}},
$$

we need to bound the products of the RHS of (36) with an arbitrary $\boldsymbol{v} \in \mathbf{H}_{0}^{1}(\Omega)$. We have:

$$
\begin{aligned}
& \frac{1}{\delta t}\left(\boldsymbol{e}_{c}^{n+1}-\boldsymbol{e}_{c}^{n}, \boldsymbol{v}\right) \leqslant \frac{1}{\delta t}\left\|\boldsymbol{e}_{c}^{n+1}-\boldsymbol{e}_{c}^{n}\right\|_{-1}\|\boldsymbol{v}\|_{1}, \quad\left\langle-v \Delta\left(\boldsymbol{e}_{c}^{n+1}\right), \boldsymbol{v}\right\rangle=v\left\|\boldsymbol{e}_{c}^{n+1}\right\|_{1}\|\boldsymbol{v}\|_{1}, \\
& \left\langle-\mathbf{R}^{n}, \boldsymbol{v}\right\rangle \leqslant\left\|\mathbf{R}^{n}\right\|_{-1}\|\boldsymbol{v}\|_{1} \leqslant C\left(\int_{t_{n}}^{t_{n+1}} t\left\|\boldsymbol{u}_{t t}\right\|_{-1}^{2} \mathrm{~d} t\right)^{1 / 2}\|\boldsymbol{v}\|_{1} .
\end{aligned}
$$

For the nonlinear terms, we use the following splitting:

$$
\begin{aligned}
& -\left(\boldsymbol{u}^{n} \cdot \nabla\right) \boldsymbol{u}^{n+1 / 2}+\left(\boldsymbol{u}\left(t_{n+1}\right) \cdot \nabla\right) \boldsymbol{u}\left(t_{n+1}\right) \\
& \quad=\left(\left(\boldsymbol{u}\left(t_{n+1}\right)-\boldsymbol{u}\left(t_{n}\right)\right) \cdot \nabla\right) \boldsymbol{u}\left(t_{n+1}\right)+\left(\boldsymbol{e}_{c}^{n} \cdot \nabla\right) \boldsymbol{u}\left(t_{n+1}\right)+\left(\boldsymbol{u}^{n} \cdot \nabla\right) \boldsymbol{e}_{c}^{n+1 / 2} .
\end{aligned}
$$

Calling I, II and III the three terms obtained after testing (38) with $v$, we have: 


$$
\begin{aligned}
& \mathrm{I} \leqslant C\left\|\boldsymbol{u}\left(t_{n+1}\right)-\boldsymbol{u}\left(t_{n}\right)\right\|_{0}\left\|\boldsymbol{u}\left(t_{n+1}\right)\right\|_{2}\|\boldsymbol{v}\|_{1} \leqslant C\left(\delta t \int_{t_{n}}^{t_{n+1}}\left\|\boldsymbol{u}_{t}\right\|_{0}^{2} \mathrm{~d} t\right)^{1 / 2}\|\boldsymbol{v}\|_{1}, \\
& \mathrm{II} \leqslant C\left\|\boldsymbol{e}_{c}^{n}\right\|_{1}\left\|\boldsymbol{u}\left(t_{n+1}\right)\right\|_{1}\|\boldsymbol{v}\|_{1} \leqslant C\left\|\boldsymbol{e}_{c}^{n}\right\|_{1}\|\boldsymbol{v}\|_{1}, \\
& \mathrm{III} \leqslant C\left\|\boldsymbol{u}^{n}\right\|_{1}\left\|\boldsymbol{e}_{c}^{n+1 / 2}\right\|_{1}\|\boldsymbol{v}\|_{1} \leqslant C\left\|\boldsymbol{e}_{c}^{n+1 / 2}\right\|_{1}\|\boldsymbol{v}\|_{1},
\end{aligned}
$$

where we have used (R1) and (23). Thus, we obtain:

$$
\begin{aligned}
\left\|r_{c}^{n+1}\right\|_{L_{0}^{2}(\Omega)} \leqslant \frac{C}{\delta t}\left\|\boldsymbol{e}_{c}^{n+1}-\boldsymbol{e}_{c}^{n}\right\|_{-1}+C & \left\{\left\|\boldsymbol{e}_{c}^{n+1}\right\|_{1}+\left\|\boldsymbol{e}_{c}^{n}\right\|_{1}+\left\|\boldsymbol{e}_{c}^{n+1 / 2}\right\|_{1}\right. \\
& \left.+\left(\int_{t_{n}}^{t_{n+1}} t\left\|\boldsymbol{u}_{t t}\right\|_{-1}^{2} \mathrm{~d} t\right)^{1 / 2}+\left(\delta t \int_{t_{n}}^{t_{n+1}}\left\|\boldsymbol{u}_{t}\right\|_{0}^{2} \mathrm{~d} t\right)^{1 / 2}\right\},
\end{aligned}
$$

which yields:

$$
\begin{aligned}
\left\|r_{c}^{n+1}\right\|_{L_{0}^{2}(\Omega)}^{2} \leqslant & \frac{C}{\delta t^{2}}\left\|\boldsymbol{e}_{c}^{n+1}-\boldsymbol{e}_{c}^{n}\right\|_{-1}^{2} \\
& +C\left\{\left\|\boldsymbol{e}_{c}^{n+1}\right\|_{1}^{2}+\left\|\boldsymbol{e}_{c}^{n}\right\|_{1}^{2}+\left\|\boldsymbol{e}_{c}^{n+1 / 2}\right\|_{1}^{2}+\int_{t_{n}}^{t_{n+1}} t\left\|\boldsymbol{u}_{t t}\right\|_{-1}^{2} \mathrm{~d} t+\delta t \int_{t_{n}}^{t_{n+1}}\left\|\boldsymbol{u}_{t}\right\|_{0}^{2} \mathrm{~d} t\right\},
\end{aligned}
$$

and (35) results from (34), the regularity properties (R3) and (R2a) (which is implied by (R2b)) of the continuous solution $\boldsymbol{u}$, and the estimates of Lemma 1 .

\section{References}

[1] J. Blasco, R. Codina, A. Huerta, A fractional-step method for the incompressible Navier-Stokes equations related to a predictor-multicorrector algorithm, Internat. J. Numer. Methods Fluids 28 (1997) 1391-1419.

[2] A.N. Brooks, T.J.R. Hughes, Streamline upwind Petrov-Galerkin formulations for convection dominated flows with particular enphasis on the incompressible Navier-Stokes equations, Comput. Methods Appl. Mech. Engrg. 32 (1982) 199-259.

[3] B. Bujanda, J.C. Jorge, Stability results for fractional step discretizations of time dependent coefficient evolutionary problems, Appl. Numer. Math. 38 (1-2) (2001) 69-86.

[4] A.J. Chorin, Numerical solution of the Navier-Stokes equations, Math. Comp. 22 (1968) 745-762.

[5] A.J. Chorin, On the convergence of discrete approximations to the Navier-Stokes equations, Math. Comp. 23 (1969) $341-353$.

[6] J.L. Cruz Soto, M.C. Calzada Canalejo, M. Marin Beltrán, E. Fernandez-Cara, A convergence result for a parallel algorithm for solving the Navier-Stokes equations, Comput. Math. Appl. 35 (4) (1998) 71-88.

[7] P. Constantin, C. Foias, Navier-Stokes Equations, University of Chicago Press, Chicago, 1988.

[8] J. Donea, S. Giuliani, H. Laval, L. Quartapelle, Finite element solution of the unsteady Navier-Stokes equations by a fractional step method, Comput. Methods Appl. Mech. Engrg. 30 (1982) 53-73.

[9] E. Fernandez-Cara, M. Marin Beltrán, The convergence of two numerical schemes for the Navier-Stokes equations, Numer. Math. 55 (1989) 33-60.

[10] V. Girault, A. Raviart, Finite Element Approximation of the Navier-Stokes Equation, Springer, New York, 1986.

[11] R. Glowinski, T.W. Pan, J. Periaux, A fictitious domain method for external incompressible viscous flow modeled by Navier-Stokes equations, Comput. Methods Appl. Mech. Engrg. 112 (1994) 133-148. 
[12] J.L. Guermond, L. Quartapelle, On the approximation of the unsteady Navier-Stokes equations by finite element projection methods, Numer. Math. 80 (1998) 207-238.

[13] J.G. Heywood, R. Rannacher, Finite element approximation of the nonstationary Navier-Stokes problem I. Regularity of solutions and second order error estimates for spatial discretization, SIAM J. Numer. Anal. 19 (2) (1982) $275-311$.

[14] J. van Kan, A second-order accurate pressure correction scheme for viscous incompressible flow, SIAM J. Sci. Statist. Comput. 7 (3) (1986) 870-891.

[15] J. Kim, P. Moin, Application of a fractional-step method to incompressible Navier-Stokes equations, J. Comput. Phys. 59 (1985) 308-323.

[16] Long-an Ying, Viscosity-splitting scheme for the Navier-Stokes equations, Numer. Methods Partial Differential Equations 7 (1991) 317-338.

[17] R. Natarajan, A numerical method for incompressible viscous flow simulation, J. Comput. Phys. 100 (1992) $384-395$.

[18] S.A. Orzag, M. Israeli, M.O. Deville, Boundary conditions for incompressible flows, J. Sci. Comput. 1 (1) (1986) $75-111$.

[19] A. Quarteroni, F. Saleri, A. Veneziani, Factorization methods for the numerical approximation of Navier-Stokes equations, Comput. Methods Appl. Mech. Engrg. 188 (2000) 505-526.

[20] J. Shen, On error estimates of projection methods for Navier-Stokes equations: First-order schemes, SIAM J. Numer. Anal. 29 (1992) 57-77.

[21] J. Shen, Remarks on the pressure error estimates for the projection method, Numer. Math. 67 (1994) 513-520.

[22] R. Temam, Une méthode d'approximations de la solution des équations de Navier-Stokes, Bull. Soc. Math. France 98 (1968) 115-152.

[23] R. Temam, Sur la stabilité et la convergence de la méthode des pas fractionaires, Ann. Mat. Pura Appl. 74 (1968) 191-380.

[24] R. Temam, Navier-Stokes Equations. Theory and Numerical Analysis, third ed., North-Holland, Amsterdam, 1984.

[25] R. Temam, Remark on the pressure boundary condition for the projection method, Theor. Comput. Fluid Dyn. 3 (1991) 181-184.

[26] L.P.J. Timmermans, P.D. Minev, F.N. van de Vosse, An approximate projection scheme for incompressible flow using spectral elements, Internat. J. Numer. Methods Fluids 22 (1996) 673-688. 